\title{
CONSTRUCTIVE POLYNOMIAL APPROXIMATION ON SPHERES AND PROJECTIVE SPACES
}

\author{
BY \\ DAVID L. RAGOZIN $\left({ }^{1}\right)$
}

\begin{abstract}
This paper contains constructive generalizations to functions defined on spheres and projective spaces of the Jackson theorems on polynomial approximation. These results, (3.3) and (4.6), give explicit methods of constructing uniform approximations to smooth functions on these spaces by polynomials, together with error estimates based on the smoothness of the function and the degree of the polynomial. The general method used exploits the fact that each space considered is the orbit of some compact subgroup, $G$, of an orthogonal group acting on a Euclidean space. For such homogeneous spaces a general result $(2.1)$ is proved which shows that a $G$-invariant linear method of polynomial approximation to continuous functions can be modified to yield a linear method which produces better approximations to $k$-times differentiable functions. Jackson type theorems (3.4) are also proved for functions on the unit ball (which is not homogeneous) in a Euclidean space.
\end{abstract}

Introduction. We previously extended the Jackson theorems to any smooth compact submanifold $M$ of a Euclidean space $\boldsymbol{E}$ (see [12]). The proofs of these theorems were not really constructive and made use of a rather ad hoc extension of a function from $M$ to some ball in $\boldsymbol{E}$. In the present paper we show how, in case $M$ is a sphere or projective space, constructive versions of these theorems can be proved which do not require us to extend functions of $M$.

We begin in $\$ 1$ by defining differentiability and other smoothness properties for functions on a homogeneous manifold $M$ in terms of the homogeneous structure of $M$. Then in $\S 2$ we show how the homogeneity of $M$ together with the Jackson theorems for $C(M)$ can be combined to prove the Jackson estimates for $C^{k}(M)$. These general results are applied to prove the Jackson estimates for spheres and balls in $\$ 3$ and for projective spaces over the reals, complexes and quaternions in $\S 4$.

A general reference for the differential geometry of homogeneous spaces of compact Lie groups which we use is Helgason [4]. However we have tried in $\S \S 3$ and 4 to be reasonably explicit so that the reader without a background in Lie

Received by the editors June 19, 1970.

AMS 1969 subject classifications. Primary 4115, 4141, 4155, 5750.

Key words and phrases. Polynomial approximation on spheres, polynomial approximation on projective spaces, Jackson estimates, invariant approximation operators, homogeneous spaces.

( ${ }^{1}$ Some of the research for this paper was supported by NSF Grants GP-9003 and GP-7461. 
theory should be able to follow the results of $\S \S 1$ and 2 , at least for the most important special cases of the spheres.

1. Smooth functions on homogeneous spaces. In this section we shall define various measures of smoothness for functions defined on a compact homogeneous submanifold $M$ of a Euclidean space $\boldsymbol{E}$. By homogeneous submanifold we mean that $M$ is the orbit of some compact subgroup $G$ of the orthogonal group of $E$. An important example which the reader may keep in mind is $M=S^{m}$, the unit sphere in $(m+1)$-space, which is the orbit of any unit vector under the action of $S O(m+1)$, the group of $(m+1)$-dimensional orthogonal matrices of determinant 1. This example is considered in more detail in $\$ 3$.

We exploit the homogeneity of $M$ to define differentiability and other smoothness properties of functions. Specifically, let $\mathfrak{g}$, the Lie algebra of $G$, be the set of all skew-symmetric operators $D$ on $\boldsymbol{E}$ (i.e. $D^{*}=-D$ where $D^{*}$ is the adjoint of $D$ ), such that $\exp t D$ is in $G$ for all $t$ in $R$. Now in terms of the action of $G$ on $C(M)$, given by $g \cdot f(m)=f\left(g^{-1} m\right)$, the space $C^{1}(M)$ of continuously differentiable functions on $M$ consists of those $f$ in $C(M)$ such that for each $D$ in $g$ there exists $D f$ in $C(M)$ with

$$
\lim _{t \rightarrow 0}\left\|t^{-1}(\exp t D \cdot f-f)-D f\right\|_{\infty}=0 .
$$

We shall use the fact that the mapping $D \mapsto D f$ is linear from $g$ to $C(M)$. The $k$ times differentiable functions on $M, C^{k}(M)$, are defined similarly.

Moduli of smoothness for functions in $C(M)$ can be defined once we have a metric on $M$ which is connected with $G$. Such a metric arises from the choice of an inner product on $g$, which is invariant under the maps $D \mapsto g D g^{-1}, g$ in $G$. One such inner product and its associated norm is given by

$$
\left(D_{1}, D_{2}\right)=\frac{1}{2} \operatorname{Tr}\left(D_{1} D_{2}^{*}\right), \quad\|D\|=(D, D)^{1 / 2},
$$

where $\operatorname{Tr}(D)$ is the trace of the operator $D$. We choose one invariant inner product and use it to define a $G$-invariant Riemannian metric on $M$ as follows. Pick a $v_{0}$ in $M$ and set $H=\left\{g\right.$ in $\left.G: g v_{0}=v_{0}\right\}$. Let $\mathfrak{h}$ be the Lie algebra of $H$ and let $\mathfrak{m}=\mathfrak{h}^{\perp}$ be the orthogonal complement of $\mathfrak{h}$ in $\mathfrak{g}$ with respect to the inner product. Then $\mathfrak{m}$ is (isomorphic to) the tangent space to $M$ at $v_{0}$ and the restriction of the inner product to $\mathfrak{m} \times \mathfrak{m}$ gives rise to an inner product on this tangent space. The action of $G$ on $M$ translates this inner product to the tangent spaces at each point of $M$ and in this way a Riemannian metric is defined. We shall measure distances on $M$ via the arclength metric, $\rho$, induced by this Riemannian metric. It can be shown, although we shall not need it, that any two such metrics, arising from different invariant inner products, are boundedly equivalent.

The first modulus of smoothness (the modulus of continuity) for $f$ in $C(M)$ is defined by

$$
\omega_{1}(f ; h)=\sup \{|f(x)-f(y)|: \rho(x, y) \leqq h\}
$$


If we introduce the notation $x_{+}, x, x_{-}$for any ordered triple of equally spaced points along a geodesic in $M$, then the second modulus of smoothness for $f$ in $C(M)$ is defined by

$$
\omega_{2}(f ; h)=\sup \left\{\left|f\left(x_{+}\right)-2 f(x)+f\left(x_{-}\right)\right|: \rho\left(x_{+}, x\right) \leqq h\right\} .
$$

To measure the moduli of smoothness for derivatives we first fix some orthonormal basis $D_{1}, \ldots, D_{j}$ for $g$. Then by induction on $k$ we introduce the following notation for $f$ in $C^{k+1}(M)$ :

$$
\omega_{r}\left(f^{(k+1)} ; h\right)=\sum_{1}^{j} \omega_{r}\left(\left(D_{i} f\right)^{(k)} ; h\right), \quad r=1,2 .
$$

(Of course $f^{(0)}=f$.) Thus $\omega_{r}\left(f^{(k)} ; h\right)$ is the sum of the $r$ th modulus of smoothness of $D^{k} f$ as $D^{k}$ ranges over all $k$-fold products of $D_{1}, \ldots, D_{j}$.

We shall need two properties of the moduli of smoothness.

Proposition 1.2. (i) If $f \in C(M)$, then $\omega_{r}(f ; d h) \leqq(1+d)^{r} \omega_{r}(f ; h), r=1,2$.

(ii) If $f \in C^{1}(M)$, then $\omega_{r}(f ; h) \leqq r\left(\sum_{1}^{j}\left\|D_{i} f\right\|_{\infty}\right) h, r=1,2$.

REMARK. 1.2 (ii) is closely related to, and will be referred to as, the mean value theorem.

Proof. Both of these follow from the fact that any geodesic from $x$ in $M$, with arclength parameter $s$, has the form $\exp -s D \cdot x$ for some $D$ in $\mathfrak{g}$ with $\|D\|=1$ $[10$, p. 52]. Then the usual facts about moduli of smoothness for the function of one variable, $g(s)=f(\exp -s D \cdot x)$, yield (i) [8, p. 47-48]. For (ii) we note that, since $g^{\prime}(s)=D f(\exp -s D \cdot x)$, the mean value theorem applied to $g$ shows that

$$
|f(\exp -s D \cdot x)-f(x)| \leqq\left\|g^{\prime}\right\|_{\infty} s \leqq\|D f\|_{\infty} s .
$$

However $D=\sum_{1}^{j} b_{i} D_{i}$ with $\sum\left|b_{i}\right|^{2}=1$. So $\left|b_{i}\right| \leqq 1$ and

$$
\|D f\|_{\infty} \leqq \sum_{1}^{j}\left|b_{i}\right|\left\|D_{i} f\right\|_{\infty} \leqq \sum_{1}^{j}\left\|D_{i} f\right\|_{\infty} .
$$

Combining these two estimates yields

$$
\omega_{1}(f ; h) \leqq\left(\sum_{1}^{j}\left\|D_{i} f\right\|_{\infty}\right) h .
$$

But $\omega_{2}(f ; h) \leqq 2 \omega_{1}(f ; h)$ so (ii) is proved for $r=1$ and 2 .

2. Invariant linear approximation operators. In this section we shall show how the homogeneity of $M$ allows us to derive Jackson type approximation theorems for functions in $C^{k}(M)$ from corresponding theorems for functions in $C(M)$. The chief tool in this derivation is a continuous linear map $T: C(M) \rightarrow C(M)$ which is $G$-invariant, i.e. such that $g \cdot T f=T(g \cdot f)$ for all $g$ in $G$. For such maps we have the following 
Theorem 2.1. Let $T: C(M) \rightarrow C(M)$ be a continuous $G$-invariant linear map. Suppose that for some $A, h>0, T$ satisfies

(i $\left.\mathrm{i}_{0}\right)$ for all $f \in C(M),\|f-T f\|_{\infty} \leqq A \omega_{r}(f ; h), r=1$ or 2.

Then ${ }_{k} T=I-(I-T)^{k+1}$ satisfies

(i $\left.i_{k}\right)$ for all $f \in C^{k}(M),\left\|f-{ }_{k} T f\right\|_{\infty} \leqq A(A r)^{k} h^{k} \omega_{r}\left(f^{(k)} ; h\right)$.

Moreover the range of ${ }_{k} T$ is included in the range of $T$.

Before the proof we need the

LEMMA 2.2. If $T: C(M) \rightarrow C(M)$ is a continuous G-invariant linear map then $T D f=D T f$ for all $f$ in $C^{1}(M)$ and all $D$ in $\mathfrak{g}$.

Proof. The continuity and invariance of $T$ justify the second and third equalities in the following chain:

$$
\begin{aligned}
T D f & =T\left(\lim _{t \rightarrow 0} t^{-1}(\exp t D \cdot f-f)\right) \\
& =\lim _{t \rightarrow 0} t^{-1}(T(\exp t D \cdot f)-T f) \\
& =\lim _{t \rightarrow 0} t^{-1}(\exp t D \cdot T f-T f)=D T f
\end{aligned}
$$

Proof of theorem. Our proof is by induction on $k$; the case $k=0$ is assumed in the theorem. For the induction step we assume $\left(i_{k}\right)$ and prove that $\left(i_{k+1}\right)$ follows. Now for $f$ in $C^{k+1}(M),\left(\mathrm{i}_{0}\right)$ and the mean value theorem, (1.2(ii)), yield

$$
\begin{aligned}
\left\|f-{ }_{k+1} T f\right\|_{\infty} & =\left\|(I-T)(I-T)^{k+1} f\right\|_{\infty} \\
& \leqq A \omega_{r}\left((I-T)^{k+1} f ; h\right) \\
& \leqq A r \cdot h \sum_{1}^{j}\left\|D_{i}(I-T)^{k+1} f\right\|_{\infty} .
\end{aligned}
$$

Hence by the previous lemma, the induction hypothesis and the definition, (1.1), of $\omega_{r}\left(f^{(k+1)} ; h\right)$, we have

$$
\begin{aligned}
\left\|f-{ }_{k+1} T f\right\|_{\infty} & \leqq A r \cdot h \sum_{1}^{j}\left\|(I-T)^{k+1} D_{i} f\right\|_{\infty} \\
& \leqq A r \cdot h \sum_{1}^{j} A(A r)^{k} h^{k} \omega_{r}\left(\left(D_{i} f\right)^{(k)} ; h\right) \\
& =A(A r)^{k+1} h^{k+1} \omega_{r}\left(f^{(k+1)} ; h\right) .
\end{aligned}
$$

Since ${ }_{k} T$ is a polynomial in $T$ without constant term the statement about ranges is clear.

The most important applications of this theorem are to problems of polynomial approximation on $M$. A polynomial of degree at most $n$ on $M$ is any member of the set $\mathscr{P}_{n}=\left\{p \in C(M): p=p_{n} \mid M\right.$ for some polynomial $p_{n} \in C(E)$ of (total) degree at most $n\}$. By the $k$ th order Jackson estimates for $M$ we mean the statement:

There exist constants $A_{k}(r), r=1,2$, such that for all $f$ in $C^{k}(M)$ and all $n$ there exist $p_{n}$ in $\mathscr{P}_{n}$ with $\left\|f-p_{n}\right\|_{\infty} \leqq A_{k}(r) n^{-k} \omega_{r}\left(f^{(k)} ; 1 / n\right)$. 
One of the main results of the quantitative theory of polynomial approximation theory is the proof of the Jackson estimates for all $k$. The case $k=0$ is crucial for the constructive proof of these estimates on a homogeneous space $M$ as we see in

COROLlary 2.3. The kth order Jackson estimates for a compact homogeneous space $M$ are true for all $k$ provided there exist constants $A(r), r=1,2$, and for each $n$ a continuous $G$-invariant linear map $T_{n}: C(M) \rightarrow \mathscr{P}_{n}$ such that for all $f$ in $C(M)$

$$
\left\|f-T_{n} f\right\|_{\infty} \leqq A(r) \omega_{r}(f ; 1 / n), \quad r=1,2 .
$$

Proof. For $f$ in $C^{k}(M)$, let $p_{n}={ }_{k} T_{n} f$ where ${ }_{k} T_{n}=I-\left(I-T_{n}\right)^{k+1}$ and apply the previous theorem. The $k$ th order Jackson estimates result with $A_{k}(r)$ $=A(r)(r A(r))^{k}$.

3. Polynomial approximation on spheres and balls. Now we consider specific examples of homogeneous manifolds for which we are able to construct $G$-invariant polynomial valued maps satisfying (2.3.1). Thus for these spaces constructive versions of the Jackson estimates will be true. In this section we study the unit sphere and, although it is not homogeneous, the unit ball in Euclidean space. In the next section we take up the projective spaces over the reals, complexes, and quaternions.

Let $M=S^{m}$ be the unit sphere in $\boldsymbol{R}^{m+1}$ with the inner product $\langle x, y\rangle$ and norm $|x|=\sqrt{ }\langle x, x\rangle$. We think of $\boldsymbol{R}^{m+1}$ as given by column vectors with respect to the standard orthonormal basis $\left\{e_{i}\right\}$. Then $S^{m}=G \cdot e_{1}$ where $G=S O(m+1)$ is the group of orthogonal matrices of determinant 1. The isotropy group, $H=\left\{g\right.$ in $\left.G: g \cdot e_{1}=e_{1}\right\}$ consists of the orthogonal matrices of the form $\left[\begin{array}{ll}1 & 0 \\ 0 & h\end{array}\right]$ where $h$ is an element in $S O(m)$. The Lie algebra, $\mathrm{g}$, consists of all skew-symmetric maps of $\boldsymbol{R}^{m+1}$ and we choose the inner product on $\mathfrak{g}$ given by $\left(D_{1}, D_{2}\right)=-(1 / 2) \operatorname{Tr}\left(D_{1} D_{2}\right)$. If $\mathfrak{h} \subseteq \mathfrak{g}$ is the Lie algebra of $H$ then $\mathfrak{m}=\mathfrak{h}^{\perp}$ consists of those maps given by matrices of the form $D_{v}=\left[\begin{array}{cc}0 & v^{*} \\ -v & 0\end{array}\right]$ with $v$ in $R^{m}$ and $\left\|D_{v}\right\|=|v|$. The geodesics through $e_{1}$ in the Riemannian metric determined by the inner product on $g$ are given by the curves $\exp -s D_{v} \cdot e_{1}=(\cos s) e_{1}+(\sin s) v$ where $|v|=1$ and $s$ is the arclength. Such a curve is just a great circle on the sphere, so by use of the $G$-action we see that the metric $\rho$ on $S^{m}$ is just the usual arclength metric on a sphere

$$
\rho(x, y)=\arccos \langle x, y\rangle, \quad x, y \text { in } S^{m} .
$$

In [9] D. J. Newman and H. Shapiro have shown how to construct operators $T_{n}: C\left(S^{m}\right) \rightarrow \mathscr{P}_{n}$ satisfying the hypothesis of Corollary 2.3 in case $r=1$. We recall their construction and show that it can be extended to the case $r=2$. Later we shall see that the same techniques work on all projective spaces.

First we give a simple method for producing $G$-invariant linear maps on $C\left(S^{m}\right)$. Let $\mu$ be normalized Lebesgue "surface" measure on $S^{m}$. This is just the natural normalized measure on $S^{m}$ associated to the $G$-invariant metric we have on $S^{m}$. Then we have the construction of 
Proposition 3.1. Let $p(t)$ be a continuous function on $[-1,1]$ and define the operator $T: C\left(S^{m}\right) \rightarrow C\left(S^{m}\right)$ by

$$
T f(x)=\int f(y) p(\langle x, y\rangle) d \mu(y), \quad x \text { in } S^{m} .
$$

Then $T$ is $G$-invariant. In fact $T$ commutes with the full orthogonal group.

Proof. This is a simple consequence of the orthogonal invariance of the measure $\mu$. Thus if $g$ is any orthogonal map

$$
\begin{aligned}
g \cdot T f(x) & =T f\left(g^{-1} \cdot x\right)=\int f(y) p\left(\left\langle g^{-1} \cdot x, y\right\rangle\right) d \mu(y) \\
& =\int f\left(g^{-1} \cdot y\right) p\left(\left\langle g^{-1} \cdot x, g^{-1} \cdot y\right\rangle\right) d \mu(y) \\
& =\int g \cdot f(y) p(\langle x, y\rangle) d \mu(y)=T(g \cdot f)(x)
\end{aligned}
$$

We remark that if $p(t)$ is a polynomial of degree at most $n$, then the range of $T$ is in $\mathscr{P}_{n}$ since $p(\langle x, y\rangle)$ is, as a function of $x$, a polynomial of degree at most $n$. Now in [9] it is shown that there exist polynomials $p_{n}(t)$ of degree at most $n$ such that

$$
\begin{aligned}
& \text { (i) } p_{n}(t) \geqq 0, \quad-1 \leqq t \leqq 1, \\
& \text { (ii) } \int p_{n}(\langle x, y\rangle) d \mu(y)=1, \quad x \text { in } S^{m}, \\
& \text { (iii) } \int(1-\langle x, y\rangle) p_{n}(\langle x, y\rangle) d \mu(y) \leqq B(m) / n^{2},
\end{aligned}
$$

where the constant $B(m)$ depends only on $m$.

When restated in terms of the polynomial-valued map $T_{n}$ associated to $p_{n}$ as in (3.1.1) these properties become

$$
\begin{aligned}
\text { (i') } & T_{n} \text { is positive, } \\
\text { (ii') } & T_{n} 1=1, \\
\text { (iii') } & T_{n}(1-\langle x, \cdot\rangle) \leqq B(m) / n^{2}, \quad x \text { in } S^{m} .
\end{aligned}
$$

We apply these to prove the main constructive polynomial approximation theorem for $S^{m}$.

THEOREM 3.3. (The Jackson estimates for $S^{m}$.) Let $T_{n}: C\left(S^{m}\right) \rightarrow \mathscr{P}_{n}$ be the operator constructed from $p_{n}$ as in (3.2.1), and let ${ }_{k} T_{n}=I-\left(I-T_{n}\right)^{k+1}$. Then there exist constants $A_{k}(r), r=1,2$, such that for all $f$ in $C^{k}\left(S^{m}\right)$ the polynomial ${ }_{k} T_{n} f$ satisfies

$$
\left\|f-{ }_{k} T_{n} f\right\|_{\infty} \leqq A_{k}(r) n^{-k} \omega_{r}\left(f^{(k)} ; 1 / n\right)
$$


Proof. Since $T_{n}$ is $G$-invariant it suffices by Corollary 2.3 to show that (3.3.1) holds when $k=0$. In [9] this is done for $r=1$. We give the simple adaptation of this Korovkin type proof to the case $r=2$.

For fixed $x$ in $S^{m}$ let $g_{x}$ be the orthogonal map given by reflection in the axis through $x$ and $-x$. Then for any $y$ in $S^{m} y, x, g_{x} \cdot y$ are always equally spaced along a geodesic (great circle). Thus

$$
\left|f(y)+f\left(g_{x} \cdot y\right)-2 f(x)\right| \leqq \omega_{2}(f ; \rho(x, y)) .
$$

To estimate $f(x)-T_{n} f(x)$ we note that since $g_{x} \cdot x=x$ we have

$$
T_{n} f(x)=g_{x}^{-1} \cdot T_{n} f(x)=T_{n}\left(g_{x}^{-1} \cdot f\right)(x) .
$$

So by $\left(3.2 .1\left(i^{\prime}\right)\right)$

$$
\begin{aligned}
\left|f(x)-T_{n} f(x)\right| & =\left|f(x) T_{n} 1-\frac{1}{2} T_{n}\left(f+g_{x}^{-1} \cdot f\right)(x)\right| \\
& =\left|\frac{1}{2} T_{n}\left(2 f(x) 1-\left(f+g_{x}^{-1} \cdot f\right)\right)(x)\right| .
\end{aligned}
$$

Now using (3.3.2) and the positivity of $T_{n}$ we see that

$$
\left|f(x)-T_{n} f(x)\right| \leqq \frac{1}{2} T_{n}\left(\omega_{2}(f ; \rho(x, \cdot))\right)(x) .
$$

But the property (1.2(i)) of $\omega_{2}(f ; h)$ yields

$$
\omega_{2}(f ; \rho(x, \cdot)) \leqq(1+n \rho(x, \cdot))^{2} \omega_{2}(f ; 1 / n) \leqq 2\left(1+n^{2} \rho^{2}(x, \cdot)\right) \omega_{2}(f ; 1 / n) .
$$

So

$$
\left|f(x)-T_{n} f(x)\right| \leqq\left(1+n^{2} T_{n}\left(\rho^{2}(x, \cdot)\right)(x)\right) \omega_{2}(f ; 1 / n) .
$$

If we use the estimate of the great circle metric $\rho$ in terms of the Euclidean metric,

$$
\rho^{2}(x, y) \leqq\left(\pi^{2} / 4\right)|x-y|^{2}=\left(\pi^{2} / 2\right)(1-\langle x, y\rangle),
$$

and apply (3.2.1(iii')) and the positivity of $T_{n}$ we get

$$
\begin{aligned}
\left|f(x)-T_{n} f(x)\right| & \leqq\left(1+n^{2} T_{n}\left(\left(\pi^{2} / 2\right)(1-\langle x, \cdot\rangle)\right)(x)\right) \omega_{2}(f ; 1 / n) \\
& \leqq\left(1+\left(B(m) \pi^{2} / 2\right)\right) \omega_{2}(f ; 1 / n) .
\end{aligned}
$$

Thus (3.3.1) holds for $k=0$ with $A_{0}(2)=\left(1+B(m) \pi^{2} / 2\right)$.

It is a simple matter to prove a Jackson-type theorem for differentiable functions on the closed unit ball, $B^{m}$, in $\boldsymbol{R}^{m}$ given the Jackson estimates for the sphere, $S^{m}$. However, since $B^{m}$ is not a homogeneous submanifold, we need certain new notations to state our results precisely. If $f$ is in $C^{k}\left(B^{m}\right)$ we shall write $\partial_{i} f$ for $\partial f / \partial x_{i}$ and if $\beta$ is an $m$-dimensional multi-index we write $\partial^{\beta} f$ for $\partial^{\beta} f / \partial x^{\beta}$. Then we set

$$
\begin{aligned}
\|f\|_{k} & =\sum_{|\beta| \leqq k}\left\|\partial^{\beta} f\right\|_{\infty}, \\
\omega(f ; h) & =\sup \{|f(x)-f(y)|:|x-y| \leqq h\}, \\
\omega\left(f^{(k)} ; h\right) & =\sum_{|\beta|=k} \omega\left(\partial^{\beta} f ; h\right) .
\end{aligned}
$$


THEOREM 3.4. (Jackson theorem for $B^{m}$.) Given $f$ in $C^{k}\left(B^{m}\right)$ there exist polynomials, $p_{n}$, with $p_{n}$ of degree at most $n$, such that

$$
\left\|f-p_{n}\right\|_{\infty} \leqq D(k, m) n^{-k}\left(n^{-1}\|f\|_{k}+\omega\left(f^{(k)} ; 1 / n\right)\right)
$$

where $D(k, m)$ is a constant which depends only on $k$ and $m$.

Proof. We follow the idea used in [9] (for the case $k=0$ of this theorem) of lifting functions on $B^{m}$ to functions on $S^{m}$ and then approximating the lifted function. So we let $\pi: S^{m} \rightarrow B^{m}$ be the projection on the first $m$ coordinates. Pick $f$ in $C^{k}\left(B^{m}\right)$. Then since $\pi$ is a $C^{\infty}$-map, $f \circ \pi$ is in $C^{k}\left(S^{m}\right)$. Now by the Jackson estimates for $S^{m}$ there exist polynomials $q_{n}\left(x_{1}, \ldots, x_{m+1}\right)$ of degree at most $n$ such that

$$
\left\|f \circ \pi-q_{n}\right\|_{\infty, s^{m}} \leqq A_{1}(k, m) n^{-k} \omega_{1}\left((f \circ \pi)^{(k)} ; 1 / n\right) .
$$

Since $f \circ \pi$ is an even function of $x_{m+1}$ we may assume $q_{n}$ is an even function of $x_{m+1}$. (If not

$$
q_{n}^{*}\left(x_{1}, \ldots, x_{m+1}\right)=\frac{1}{2}\left(q_{n}\left(x_{1}, \ldots, x_{m+1}\right)+q_{n}\left(x_{1}, \ldots, x_{m},-x_{m+1}\right)\right)
$$

is a polynomial, even in $x_{m+1}$, satisfying the same inequality as $q_{n}$.) Thus $x_{m+1}$ occurs only to even powers in $q_{n}$, so the function

$$
p_{n}\left(x_{1}, \ldots, x_{m}\right)=q_{n}\left(x_{1}, \ldots, x_{m},\left(1-\left(x_{1}^{2}+\cdots+x_{m}^{2}\right)\right)^{1 / 2}\right)
$$

is a polynomial of degree at most $n$ on $B^{m}$. Now on $S^{m}, x_{m+1}^{2}=1-\left(x_{1}^{2}+\cdots+x_{m}^{2}\right)$, so $\left\|f-p_{n}\right\|_{\infty, B^{m}}=\left\|f \circ \pi-q_{n}\right\|_{\infty, s^{m}}$. As a consequence of Lemma 3.5 (see below) we have

$$
\begin{aligned}
\left\|f-p_{n}\right\|_{\infty, B^{m}} & \leqq A_{1}(k, m) n^{-k} \omega_{1}\left((f \circ \pi)^{(k)} ; 1 / n\right) \\
& \leqq A_{1}(k, m) C(k, m) n^{-k}\left\{n^{-1}\|f\|_{k}+\omega\left(f^{(k)} ; 1 / n\right)\right\} .
\end{aligned}
$$

LEMMA 3.5. There exists a constant $C(k, m)$ such that

$$
\omega_{1}\left((f \circ \pi)^{(k)} ; h\right) \leqq C(k, m)\left\{h\|f\|_{k}+\omega\left(f^{(k)} ; h\right)\right\}
$$

for all $f$ in $C^{k}\left(B^{m}\right)$.

Proof. In order to define $\omega_{1}\left((f \circ \pi)^{(k)} ; h\right)$ we had to choose an orthonormal basis for the Lie algebra of $S O(m+1)$. We assume the basis chosen consisted of those skew-symmetric maps $D_{i j}, 1 \leqq i<j \leqq m+1$, given, respectively, by matrices with 1 in the $i$ th row, $j$ th column, -1 in the $j$ th row, $i$ th column and zeros elsewhere. Then an explicit calculation shows that

$$
\exp t D_{i j} \cdot(f \circ \pi)\left(x_{1}, \ldots, x_{m+1}\right)=f \circ \pi\left(y_{1}, \ldots, y_{m+1}\right)
$$

where

$$
\begin{aligned}
y_{l} & =x_{l}, & & l \neq i, j, \\
& =x_{i} \cos t-x_{j} \sin t, & & l=i, \\
& =x_{i} \sin t+x_{j} \cos t, & & l=j .
\end{aligned}
$$


Hence

$$
\begin{aligned}
D_{i j}(f \circ \pi)(x) & =\left.(d / d t)\left(\exp t D_{i j} \cdot(f \circ \pi)\right)(x)\right|_{t=0} \\
& =x_{i} \partial_{j} f(\pi(x))-x_{j} \partial_{i} f(\pi(x))
\end{aligned}
$$

where $\partial_{m+1} f=0$. Iteration of this calculation shows that if $D^{k}$ is any product of $k$ of the $D_{i j}$ then $D^{k}(f \circ \pi)(x)$ is a sum of terms of the form $p(x) \partial^{\beta} f(\pi(x)),|\beta| \leqq k$, where $p(x)$ is a polynomial. For each such term the mean value theorem in $\boldsymbol{R}^{m+1}$ yields

$$
\begin{aligned}
\mid p(x) \partial^{\beta} f(\pi(x))-p(y) & \partial^{\beta} f(\pi(y)) \mid \\
& \leqq|p(x)-p(y)|\left|\partial^{\beta} f(\pi(x))\right|+|p(x)|\left|\partial^{\beta} f(\pi(x))-\partial^{\beta} f(\pi(y))\right| \\
& \leqq\|p\|_{1}|x-y|\left\|\partial^{\beta} f\right\|_{\infty}+\|p\|_{\infty}\left\|\partial^{\beta} f\right\|_{1}|\pi(x)-\pi(y)| \quad \text { if }|\beta|<k, \\
& \leqq\|p\|_{1}|x-y|\left\|\partial^{\beta} f\right\|_{\infty}+\|p\|_{\infty} \omega\left(\partial^{\beta} f ;|\pi(x)-\pi(y)|\right) \quad \text { if }|\beta|=k .
\end{aligned}
$$

Hence summing these terms and using the fact that $|\pi(x)-\pi(y)| \leqq|x-y| \leqq \rho(x, y)$ we get

$$
\omega_{1}\left(D^{k}(f \circ \pi) ; \rho(x, y)\right) \leqq C\left\{\rho(x, y)\|f\|_{k}+\omega\left(f^{(k)} ; \rho(x, y)\right)\right\}
$$

where $C$, through the polynomials $p$, depends only on $k$ and $m$. Now we sum over all such $D^{k}$ to get the estimate of the lemma.

4. Polynomial approximation on projective spaces. To apply our basic techniques we must realize each projective space as an orbit of some compact subgroup of an orthogonal group acting on a Euclidean space. We follow Tai [14] in developing such a realization.

Let $\boldsymbol{F}$ be any one of the (skew) fields

$$
\begin{aligned}
& \boldsymbol{Q}=\left\{x_{0}+x_{1} i+x_{2} j+x_{3} k: x_{l} \in \boldsymbol{R}\right\}, \text { the quaternions, } \\
& \boldsymbol{C}=\left\{x \in \boldsymbol{Q}: x_{2}=x_{3}=0\right\}, \text { the complexes, } \\
& \boldsymbol{R}=\left\{x \in \boldsymbol{Q}: x_{1}=x_{2}=x_{3}=0\right\}, \text { the reals. }
\end{aligned}
$$

Then for $x$ in $F$ we set $\operatorname{Re} x=x_{0}$ and define the conjugate of $x$ by $\bar{x}=x_{0}-x_{1} i-x_{2} j$ $-x_{3} k . F^{m+1}$ is the right vector space of $(m+1)$ by 1 matrices over $\boldsymbol{F}$. We norm $\boldsymbol{F}^{m+1}$ by $|x|^{2}=x^{*} x$ where ${ }^{*}$ denotes the conjugate transpose. Any $(m+1)$ by $(m+1)$ matrix over $\boldsymbol{F}$ acts as an $\boldsymbol{F}$-linear map on $\boldsymbol{F}^{m+1}$ by multiplication on the left. We shall use the fact that the trace of such a matrix, $\operatorname{Tr} A=\sum a_{i i}$, where $A=\left[a_{i j}\right]$, satisfies $\operatorname{Re} \operatorname{Tr}(A B)=\operatorname{Re} \operatorname{Tr}(B A)$. This is easily verified by writing $A=A_{0}+A_{1} i$ $+A_{2} j+A_{3} k$ with $A_{l}$ a matrix over $\boldsymbol{R}$.

The space of all square matrices of size $m+1$ is a Euclidean space with respect to the inner product $\langle A, B\rangle=\operatorname{Re} \operatorname{Tr}\left(A B^{*}\right)$. The unitary matrices $\mathscr{U}=\left\{U: U^{*} U=I\right\}$ act as orthogonal maps via the adjoint action $U(A)=U A U^{*}, U \in \mathscr{U}$, and the hermitian matrices $E=\left\{A: A=A^{*}\right\}$ form an invariant subspace under this action. If $\mathscr{T}$ is the homomorphism of $\mathscr{U}$ into the orthogonal group of $\boldsymbol{E}$ induced by the adjoint action, we let $G$ denote the identity component of $\mathscr{T}(\mathscr{U})$. 
Now we realize a projective space over $\boldsymbol{F}$ as an orbit of $\boldsymbol{G}$ acting on $\boldsymbol{E}$. Specifically we set $A_{0}=\left[\begin{array}{ll}1 & 0 \\ 0 & 0_{m}\end{array}\right]$ and let $d=\operatorname{dim}_{R} F$. Then we define the $d m$-dimensional projective space over $\boldsymbol{F}$ by

$$
\boldsymbol{P}^{d m}(\boldsymbol{F})=\left\{U\left(A_{0}\right): \mathscr{T}(U) \in G\right\} .
$$

If $x$ in $F^{m+1}$ is the first column of $U$ then $U\left(A_{0}\right)=U A_{0} U^{*}=x x^{*}$. Now any $x$ in $F^{m+1}$ with $|x|=1$ can occur as the first column of a unitary map $U$ with $\mathscr{T}(U)$ in $G$, so $\boldsymbol{P}^{d m}(\boldsymbol{F})$ is the image of the unit sphere in $\boldsymbol{F}^{m+1}$ under the map $x \mapsto x x^{*}$. Since $x x^{*}=y y^{*}$ exactly when $x=y a$ for some $a$ in $F$ with $|a|=1$, we see that our definition of the projective spaces is equivalent to the more usual definition in terms of factoring the unit sphere in $\boldsymbol{F}^{m+1}$. From this latter definition it is clear that the real dimension of the homogeneous submanifold $\boldsymbol{P}^{d m}(\boldsymbol{F})$ is $d m$.

We identify the Lie algebra of $G$ as follows. A skew-symmetric matrix $D$, i.e. $D^{*}=-D$, gives rise to a skew-symmetric operator on $\boldsymbol{E}$ according to the definition

$$
D(A)=D A-A D, \quad A \in \boldsymbol{E} .
$$

Via this action the Lie algebra of $G$ is given by

$$
\mathfrak{g}=\left\{D: D^{*}=-D \text { and } \operatorname{Tr} D=0\right\} .
$$

We endow $g$ with the inner product induced by the restriction of the $\mathscr{U}-$, hence, $G$-invariant inner product on all matrices. The orthogonal complement in $\mathfrak{g}$ of the Lie algebra of the isotropy subgroup of $A_{0}, H=\left\{\mathscr{T}(U) \in G: U A_{0} U^{*}=A_{0}\right\}$, is the set

$$
\mathfrak{m}=\left\{D_{v}=\left[\begin{array}{cc}
0 & -v^{*} \\
v & 0
\end{array}\right]: v \in \boldsymbol{F}^{m}\right\}
$$

The inner product restricted to $m$ induces a Riemannian metric $\rho$ on $\boldsymbol{P}^{d m}(\boldsymbol{F})$. We summarize certain properties of this metric in

Lemma 4.1. For $A, B$ in $\boldsymbol{P}^{d m}(\boldsymbol{F})$,

(i) $\sqrt{ } 2 \rho(A, B)=\arccos (2\langle A, B\rangle-1)$,

(ii) $\rho(A, B) \leqq(\pi / 2)\|A-B\|$,

(iii) $\operatorname{Max}\{\rho(A, B)\}=\pi / \sqrt{ } 2$.

Proof. By [10, p. 52] any geodesic from $A_{0}$ has the form

$$
f_{v}(t)=\exp t D_{v} \cdot A_{0}=\left[\begin{array}{cc}
\cos ^{2} t|v| & \frac{\sin 2 t|v|}{2|v|} v^{*} \\
\frac{\sin 2 t|v|}{2|v|} v & \frac{\sin ^{2} t|v|}{|v|^{2}} v v^{*}
\end{array}\right]
$$

for some $D_{v}$ in $\mathrm{m}$. Now as $v$ varies over $|v|<\pi / 2$ the points $f_{v}(1)$ are distinct. So for $|v| \leqq \pi / 2, f_{v}(t)$ is a minimal geodesic from $A_{0}$ to $f_{v}(1)$. Thus

$$
\begin{aligned}
\rho^{2}\left(A_{0}, f_{v}(1)\right) & =\left\|D_{v}\right\|^{2}=2|v|^{2}=(1 / 2)\left(\arccos \left(2 \cos ^{2}|v|-1\right)\right)^{2} \\
& =(1 / 2)\left(\arccos \left(2\left\langle A_{0}, f_{v}(1)\right\rangle-1\right)\right)^{2}, \quad|v| \leqq \pi / 2 .
\end{aligned}
$$


Since the points $f_{v}(1),|v| \leqq \pi / 2$, cover $\boldsymbol{P}^{d m}(\boldsymbol{F})$ the homogeneity of the space and the invariance of $\rho$ and $\langle A, B\rangle$ yield (i) and (iii). For (ii) we note that since $\|A\|=1$ $=\|B\|$ we have

$$
\begin{aligned}
\|A-B\|^{2} & =2-2\langle A, B\rangle=1-(2\langle A, B\rangle-1) \\
& =1-\cos (\sqrt{ } 2 \rho(A, B))=2 \sin ^{2}(1 / \sqrt{ } 2) \rho(A, B), \quad \rho(A, B) \leqq(\pi / \sqrt{ } 2) .
\end{aligned}
$$

Now (ii) follows by a standard estimate for $\sin t$.

We remark that as a consequence of (4.1(i)) the metric $\rho$ is invariant not only under $G$ but also under any orthogonal map of $\boldsymbol{E}$ which preserves $\boldsymbol{P}^{d m}(\boldsymbol{F})$.

Now we construct $G$-invariant linear operators on $C\left(\boldsymbol{P}^{d m}(\boldsymbol{F})\right)$ just as we did on the spheres. Let $\mu$ be the normalized Riemann measure on $\boldsymbol{P}^{d m}(\boldsymbol{F})$ associated to the Riemannian metric. This measure is invariant under all orthogonal maps of $\boldsymbol{E}$ which leave $\boldsymbol{P}^{d m}(\boldsymbol{F})$ invariant. Hence the proof of the following proposition is the same as that of Proposition 3.1.

Proposition 4.2. Let $p(t)$ be a continuous function on $[-1,1]$ and define the operator T: $C\left(\boldsymbol{P}^{d m}(\boldsymbol{F})\right) \rightarrow C\left(\boldsymbol{P}^{d m}(\boldsymbol{F})\right)$ by

$$
T f(A)=\int f(B) p(2\langle A, B\rangle-1) d \mu(B) .
$$

Then $T$ is $G$-invariant. In fact $T$ is invariant under any orthogonal map of $\boldsymbol{E}$ which leaves $\boldsymbol{P}^{d m}(\boldsymbol{F})$ invariant.

Proof. We need only to remark that $T f$ is well defined since $-1 \leqq 2\langle A, B\rangle-1 \leqq 1$ by the previous lemma.

As in the case of the spheres we remark that if $p(t)$ is a polynomial of degree at most $n$ then the range of $T$ is included in $\mathscr{P}_{n}$ since $p(2\langle A, B\rangle-1)$ is, as a function of $A$, a polynomial on $\boldsymbol{E}$ of degree at most $n$. Hence to parallel our proof of the Jackson estimates for $S^{m}$ we must find polynomials $p_{n}$ of degree at most $n$ satisfying

(i) $p_{n}(t) \geqq 0, \quad-1 \leqq t \leqq 1$,

(ii) $\int p_{n}(2\langle A, B\rangle-1) d \mu(B)=1, \quad A$ in $\boldsymbol{P}^{d m}(\boldsymbol{F})$,

(iii) $\int(2-2\langle A, B\rangle) p_{n}(2\langle A, B\rangle-1) d \mu(B) \leqq B(m, d) / n^{2}$, where the constant $B(m, d)$ depends only on $m$ and $d$.

To find such polynomials we first show how to compute integrals such as those occurring above.

LEMMA 4.4. If $p(t)$ is any continuous function on $[-1,1]$, then

$$
\int p(2\langle A, B\rangle-1) d \mu(B)=c \int_{-1}^{1} p(t)(1-t)^{\alpha}(1+t)^{\beta} d t
$$

where $\alpha=(d m-2) / 2, \beta=(d-2) / 2$ and $1 / c=\int_{-1}^{1}(1-t)^{\alpha}(1+t)^{\beta} d t$. 
Proof. The function $p(2\langle A, B\rangle-1)=p(\cos \sqrt{ } 2 \rho(A, B))$ depends only on the distance of $B$ from $A$. For any such radial function $f(B)=g(\rho(A, B))$ if we choose polar coordinates about $A$ we have

$$
\int f(B) d \mu(B)=\int_{0}^{\pi / \sqrt{ } 2} g(r) A(r) d r
$$

where $A(r)$ is the "area" of the sphere (in $\boldsymbol{P}^{d m}(\boldsymbol{F})$ ) about $A$ of radius $r$. Now Helgason [4, p. 171] computes that $A(r)=c^{\prime} \sin ^{m d-d}(r / \sqrt{ } 2) \sin ^{d-1}(\sqrt{ } 2 r)$ for some constant $c^{\prime}$. If we use this, set $g(r)=p(\cos \sqrt{ } 2 r)$ and make the change of variable $t=\cos \sqrt{ } 2 r$, then we get the desired result.

The measure $(1-t)^{\alpha}(1+t)^{\beta} d t$ on $[-1,1]$ which arose in this lemma is closely connected with the classical Jacobi polynomials. We follow Newman and Shapiro [9] and exploit this connection to find polynomials satisfying (4.3).

Lemma 4.5. Let $P_{n}^{(\alpha, \beta)}(t)$ be the Jacobi polynomial of degree $n$, where $\alpha=(d m-2) / 2, \beta=(d-2) / 2$. Define $p_{n}$ by

$$
p_{2 n}(t)=p_{2 n+1}(t)=a\left(p_{n+1}^{(\alpha, \beta)}(t) /\left(t-\lambda_{n+1}^{(\alpha, \beta)}\right)\right)^{2}
$$

where $\lambda_{n+1}^{(\alpha, \beta)}$ is the largest root of $P_{n+1}^{(\alpha, \beta)}(t)$ and $a$ is chosen to make

Then

$$
c \int_{-1}^{1} p_{2 n}(t)(1-t)^{\alpha}(1+t)^{\beta} d t=1
$$

$$
c \int_{-1}^{1}(1-t) p_{2 n}(t)(1-t)^{\alpha}(1+t)^{\beta} d t=1-\lambda_{n+1}^{(\alpha, \beta)} .
$$

Moreover, the polynomials $p_{n}$ satisfy (4.3).

Proof. We use the Gaussian quadrature formula for $\int_{-1}^{1} f(t)(1-t)^{\alpha}(1+t)^{\beta} d t$ based on the roots of $P_{n+1}^{(\alpha, \beta)}[13$, p. 47]. This formula is exact for polynomials of degree at most $2 n+1$, so in particular it is exact for both $p_{2 n}$ and $(1-t) p_{2 n}(t)$. But $p_{2 n}$ vanishes at all but the largest root of $P_{n+1}^{(\alpha, \beta)}$, so the one nonzero term in the quadrature formula shows

and

$$
c \int_{-1}^{1} p_{2 n}(t)(1-t)^{\alpha}(1+t)^{\beta} d t=b p_{2 n}\left(\lambda_{n+1}^{(\alpha, \beta)}\right)=1
$$

$$
c \int_{-1}^{1}(1-t) p_{2 n}(t)(1-t)^{\alpha}(1+t)^{\beta} d t=b\left(1-\lambda_{n+1}^{(\alpha, \beta)}\right) p_{2 n}\left(\lambda_{n+1}^{(\alpha, \beta)}\right)=1-\lambda_{n+1}^{(\alpha, \beta)} .
$$

To see that (4.3) is satisfied we note that $p_{n} \geqq 0$ and the normalization constant $a$ has been chosen so as to make (4.3(ii)) hold. For (4.3(iii)) we use the estimates for the zeros of the Jacobi polynomials contained in Szegö [13, p. 236]

$$
\lambda_{n}^{(\alpha, \beta)}=\cos \left(n^{-1}(\pi+O(1))\right)
$$

where $O(1)$ is uniformly bounded for all values of $n$. Thus $\left(1-\lambda_{n+1}^{(\alpha, \beta)}\right)=O\left(1 / n^{2}\right)$. So the previous lemma shows (4.3(iii)) holds, since $2-2\langle A, B\rangle=1-(2\langle A, B\rangle-1)$. 
Now it is a simple matter to prove the main constructive polynomial approximation theorem for $\boldsymbol{P}^{d m}(\boldsymbol{F})$.

THEOREM 4.6. (The Jackson estimates for projective spaces.) Let $T_{n}: C\left(\boldsymbol{P}^{d m}(\boldsymbol{F})\right) \rightarrow$ $\mathscr{P}_{n}$ be the operator constructed from $p_{n}$ as in (4.2.1) and let ${ }_{k} T_{n}=I-\left(I-T_{n}\right)^{k+1}$. Then there exist constants $A_{k}(r), r=1,2$, such that for all $f$ in $C^{k}\left(\boldsymbol{P}^{d m}(\boldsymbol{F})\right)$ the polynomial ${ }_{k} T_{n} f$ satisfies

$$
\left\|f-{ }_{k} T_{n} f\right\|_{\infty} \leqq A_{k}(r) n^{-k} \omega_{r}\left(f^{(k)} ; 1 / n\right)
$$

Proof. Since $T_{n}$ is $G$-invariant it suffices to show that (4.6.1) holds when $k=0$. It also suffices to consider $r=2$ since $\omega_{2}(f ; h) \leqq 2 \omega_{1}(f ; h)$. The proof now proceeds exactly as in Theorem 3.3, up to (3.3.3), modulo the following observation. For any point $\boldsymbol{A}$ in $\boldsymbol{P}^{d m}(\boldsymbol{F})$ there is an orthogonal map $g_{\boldsymbol{A}}$ of $\boldsymbol{E}$, leaving $\boldsymbol{P}^{d m}(\boldsymbol{F})$ invariant, such that $B, A, g_{A} \cdot B$ are equally spaced along a geodesic for any $B$ in $\boldsymbol{P}^{d m}(\boldsymbol{F})$. (If $A=A_{0}$ this is just the map given by

$$
\left[\begin{array}{cc}
s & w^{*} \\
w & X
\end{array}\right] \mapsto\left[\begin{array}{cc}
s & -w^{*} \\
-w & X
\end{array}\right]
$$

$s$ in $\boldsymbol{R}, w$ in $\boldsymbol{F}^{m}$, and $X$ an hermitian matrix of size $m$.)

From (3.3.3) the proof is completed by using the comparison of metrics contained in (4.1(ii)).

5. Additional remarks, historical notes and open questions. The techniques used for the projective spaces can be applied to give constructive versions of the Jackson estimates for $\boldsymbol{P}^{16}$ (Cay). The necessary realizations in Euclidean space and formulae for geodesics, metrics and measures can be derived (at some length) from Tai [14] and Helgason [4], [5].

Previous work on the constructive versions of the Jackson estimates for spheres can be found for $S^{2}, k=0$, in Gronwall [3], and for $k$ arbitrary, in Kušnirenko [7] and Džafarov [2]; for $S^{m}, k=0$, in Newman and Shapiro [9]; for $S^{3}, k$ arbitrary, in Ragozin [11]. Such estimates for the multi-dimensional tori are well known (see [8, pp. 89-90]), while Sun Kung [6] has similar results for the unitary groups for $k=0$. Converse or Bernstein type theorems are proved for all compact homogeneous spaces in [12].

We first used the method presented in $\S 2$ in [11] after analyzing the usual inductive proof (e.g. [1, p. 146]) of the Jackson theorems on $S^{1}$. It was only after that work that we realized this method could be applied not only to compact groups but also to homogeneous spaces of these groups as well.

We list several open problems. First, can constructive proofs of the Jackson theorems be given for all homogeneous spaces of compact Lie groups? We suspect the answer is yes but know of no positive results other than those mentioned 
above $\left({ }^{2}\right)$. Another is whether for a particular space, say $S^{m}$, the constants $A_{k}(r)$ can be replaced by a single constant $A(r)$ which is independent of $k$. That this is so for $S^{1}$ is the celebrated result of Favard and Ahiezer-Krein. Finally we mention the problem of discovering exactly how, on the balls $B^{m}$, the error in the best polynomial approximation depends on the distance to the boundary of $B^{m}$.

\section{REFERENCES}

1. E. W. Cheney, Introduction to approximation theory, McGraw-Hill, New York, 1966. MR 36 \#5568.

2. A. S. Džafarov, On the order of the best approximations of the functions continuous on the unit sphere by means of finite spherical sums, Proc. Second All-Union Conference (Baku, 1962), Studies of Contemporary Problems in Constructive Theory of Functions, Izdat. Akad. Nauk Azerbaĭdžan. SSR, Baku, 1965, pp. 46-52. (Russian) MR 33 \#6227.

3. T. H. Gronwall, On the degree of convergence of Laplace series, Trans. Amer. Math. Soc. 15 (1914), 1-30.

4. S. Helgason, Differential geometry and symmetric spaces, Pure and Appl. Math., vol. XII, Academic Press, New York, 1962. MR 26 \#2986.

5. - The Radon transform on Euclidean spaces, compact two-point homogeneous spaces, and Grassmann manifolds, Acta Math. 113 (1965), 153-180. MR 30 \#2530.

6. Sun Kung, Fourier analysis on unitary groups. IV: On the Peter-Weyl theorem, Acta Math. Sinica 13 (1963), 323-331 = Chinese Math.-Acta 4 (1964), 351-359. MR 31 \#4861.

7. G. G. Kušnirenko, The approximation of functions defined on the unit sphere by finite spherical sums, Naučn. Dokl. Vysš. Skoly Fiz.-Mat. Nauki 1958, No. 4, 47-53. (Russian) MR $26 \# 530$.

8. G. G. Lorentz, Approximation of functions, Holt, Rinehart and Winston, New York, 1966. MR 35 \#4642.

9. D. J. Newman and H. S. Shapiro, Jackson's theorem in higher dimensions (With discussion), Proc. Conference on Approximation Theory (Oberwolfach, 1963), Birkhäuser, Basel, 1964, pp. 208-219. MR 32 \#310.

10. K. Nomizu, Invariant affine connections on homogeneous spaces, Amer. J. Math. 76 (1954), 33-65. MR 15, 468.

11. D. L. Ragozin, Approximation theory on $S U(2)$, J. Approximation Theory 1 (1968), 464-475. MR 39 \#3214.

12. - Polynomial approximation on compact manifolds and homogeneous spaces, Trans. Amer. Math. Soc. 150 (1970), 41-53.

13. G. Szegö, Orthogonal polynomials, Amer. Math. Soc. Colloq. Publ., vol. 23, Amer. Math. Soc., Providence, R. I., 1959. MR 21 \#5029.

14. S. S. Tai, Minimum imbeddings of compact symmetric spaces of rank one, J. Differential Geometry 2 (1968), 55-66. MR 37 \#6950.

University of Washington, Seattle, Washington 98105

$\left({ }^{2}\right)$ Added in proof. $\mathrm{H}$. Johnen has recently given constructive proofs of the Jackson estimates for the unitary groups based on the work in [6]. He has also announced that similar proofs can be given for the rotation, special unitary and symplectic groups. (See H. Johnen, Best approximation on the unitary group, Proceedings of the Conference on Hilbert Space Operators and Operator Algebras, Tihany (1970).) 